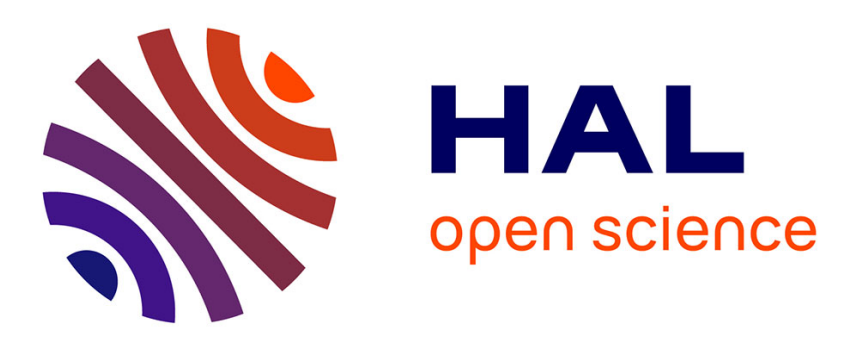

\title{
A Spiking Neural Network for Gas Discrimination using a Tin Oxide Sensor Array
}

Maxime Ambard, Bin Guo, Dominique Martinez, Amine Bermak

\section{To cite this version:}

Maxime Ambard, Bin Guo, Dominique Martinez, Amine Bermak. A Spiking Neural Network for Gas Discrimination using a Tin Oxide Sensor Array. 4th IEEE International Symposium on Electronic Design, Test \& Applications - DELTA 2008, Jan 2008, Hong-Kong, Hong Kong SAR China. inria00401777

\section{HAL Id: inria-00401777 https://hal.inria.fr/inria-00401777}

Submitted on 6 Jul 2009

HAL is a multi-disciplinary open access archive for the deposit and dissemination of scientific research documents, whether they are published or not. The documents may come from teaching and research institutions in France or abroad, or from public or private research centers.
L'archive ouverte pluridisciplinaire HAL, est destinée au dépôt et à la diffusion de documents scientifiques de niveau recherche, publiés ou non, émanant des établissements d'enseignement et de recherche français ou étrangers, des laboratoires publics ou privés. 


\title{
A Spiking Neural Network for Gas Discrimination using a Tin Oxide Sensor Array
}

\author{
Maxime Ambard ${ }^{1}$, Bin Guo ${ }^{2}$, Dominique Martinez ${ }^{1}$, Amine Bermak ${ }^{2}$ \\ ${ }^{1}$ CORTEX Group, LORIA-INRIA, Nancy, France. Email: ambard@loria.fr \\ ${ }^{2}$ Electronic and Computer Engineering Department, Hong Kong University of Science and Technology \\ Clear Water Bay, Kowloon, Hong Kong. Email: guobin@ust.hk
}

\begin{abstract}
We propose a bio-inspired signal processing method for odor discrimination. A spiking neural network is trained with a supervised learning rule so as to classify the analog outputs from a monolithic $4 \times 4$ tin oxide gas sensor array implemented in our in-house $5 \mu \mathrm{m}$ process. This scheme has been sucessfully tested on a discrimination task between 4 gases (hydrogen, ethanol, carbon monoxide, methane). Performance compares favorably to the one obtained with a common statistical classifier. Moreover, the simplicity of our method makes it well suited for building dedicated hardware for processing data from gas sensor arrays.
\end{abstract}

Keywords: Tin Oxide, Gas Sensor Array, Spike Timing Computation, Supervised Learning

\section{INTRODUCTION}

$\mathbf{T}$ HE electronic nose (EN) systems based on integrated gas sensor arrays have attracted more and more interest during the past two decades [1], [2]. $\mathrm{SnO}_{2}$-based gas sensing films are commonly used for such applications because of a number of advantages including cost effective, high sensitivity to various gases and relative compatibility with standard CMOS fabrication processes [3]. The multi-dimensional output of such gas sensor array demands specific algorithms. The high performance of biological systems to discriminate odors from multi-dimensional inputs led to an exciting new area of bioinspired algorithms.

The olfactory system anatomy of vertebrates (resp. insects) can be separated in three major parts (for more details see [4] [5]). The olfactory epithelium (resp. the insect antennae) consists of different families of Olfactory Receptor Neurons (ORNs), which transduce the molecular acivity into electrical signals. This information converges to a recurrent neural network, the Olfactory Bulb (OB) (resp. the Antennal Lobe $(A L)$ ). Principals cells of this network relay information to higher neural structures such as the piriform cortex (resp. the mushroom body).

It is well known that the different families of ORNs have broadly overlapping tuning profiles related to the molecular quality [6]. However, the neural activity in the piriform cortex (resp. the mushroom body) is more spatially differentiated with respect to the molecular species than the ORNs [7] (resp. [8]). Thus, the olfactory information seems to be transformed from a pattern of relative activity rates in ORNs to a pattern

This work was sponsored by (HKUST 6162/04E) from the Research Grant Council of Hong Kong, by INRIA (Institut National de Recherche en Informatique et Automatique) and by the French consulate, Procore Grant Ref: F-HK19/05T-II. of spatial neural activation in the piriform cortex (resp. the mushroom body).

The $\mathrm{OB}$ and the AL, which are networks of highly interconnected excitatory and local inhibitory neurons, play a key role in the odor information transfer. One of their major characteristics is to present global oscillatory activities. In the OB it has been shown that the rate of inhibitory event reception is correlated to the phase of the global oscillation [9]. This phasic inhibition, depending on its temporal distribution, has been described to act as a "reset mechanism" increasing the neural code reliability [10].

In this paper we propose a bio-inspired algorithm based on artificial neural network with periodic reset mechanism and spike timing pattern recognition to transform and decode the information from gas sensors into spatial neural activation for odor discrimination. Section II demonstrates a $4 \times 4$ gas sensor array with Micro-hotplate (MHP) structures using surface micro-machining process suitable for large dimension arrays. Section III explains in details the bio-inspired algorithm. Section IV presents the discrimination results and provides comparison with Support Vector Machine (SVM). Section V concludes this paper.

\section{SENSOR ARRAY INTEGRATION AND GAS ANALYSIS}

Tin oxide gas sensors are widely used for gas discrimination applications. The sensing mechanism of tin oxide gas sensors is based upon a chemical reaction at the surface of the sensing film.

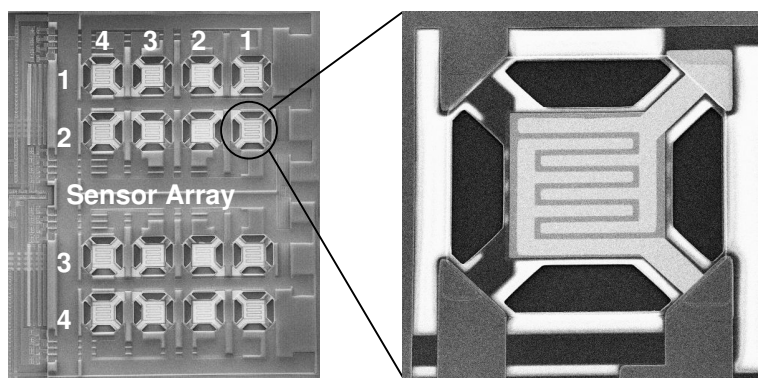

Fig. 1. (left) Microphotograph of the fabricated monolithic integrated $4 \times 4$ tin oxide gas sensor array. (right) One sensor element.

After exposure to the target gas, the resistance of the sensor is affected. The change in resistance is due to irreversible reactions between the analyte and the oxygen-derived compound such as $\mathrm{O}^{-}, \mathrm{O}_{2}^{-}$, and $\mathrm{O}^{2-}$ on the tin oxide surface [11]. The reaction is slow at room temperature, thus the tin oxide 


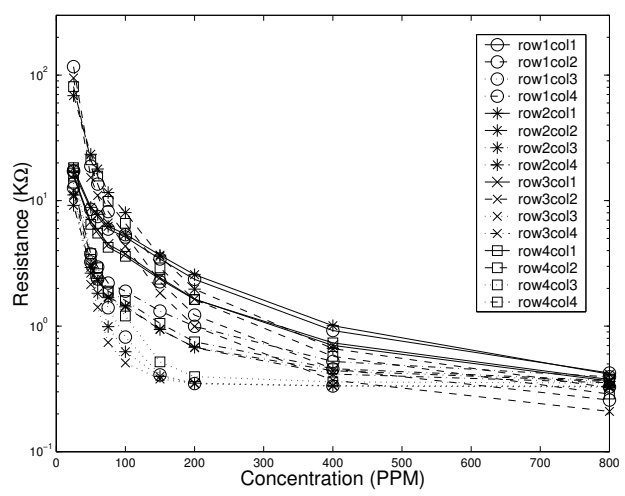

a.

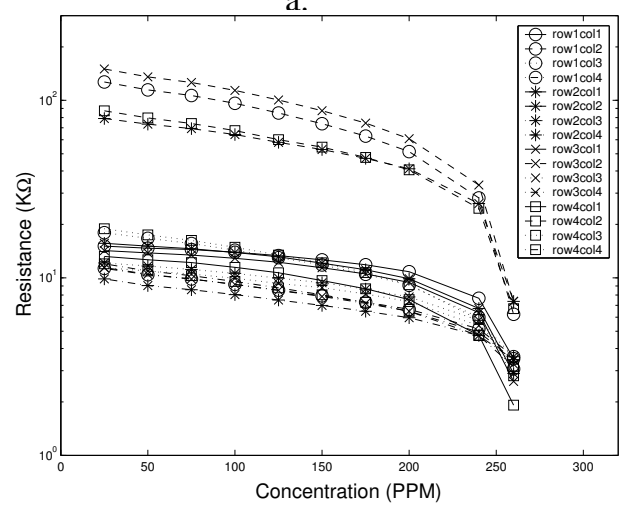

c.

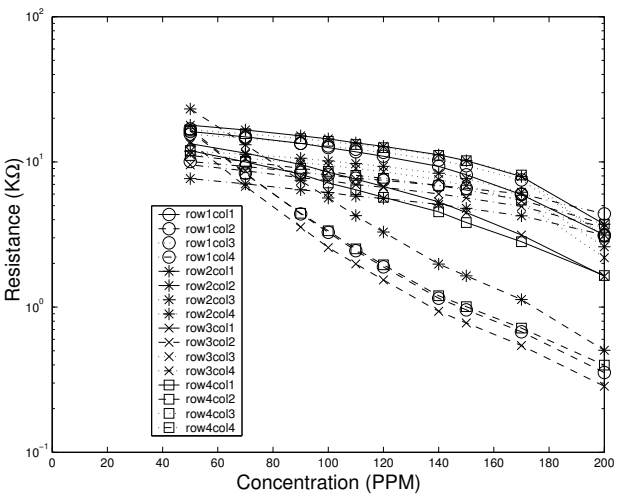

b.

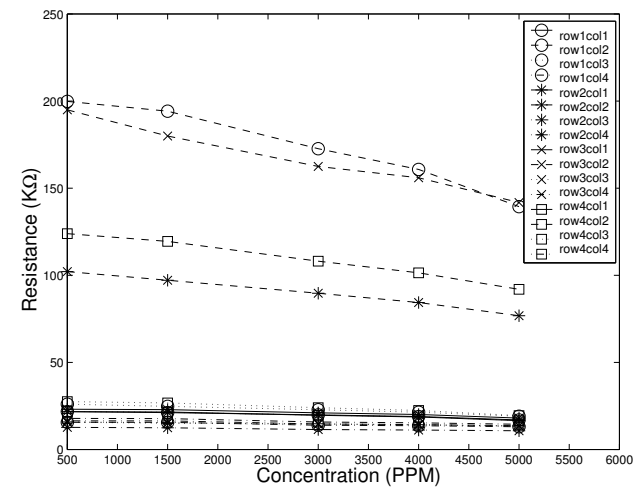

d.

Fig. 2. The sensor array's response to (a) Hydrogen (b) Ethanol (c) Carbon monoxide and (d) Methane.

gas sensor operates at high temperature, typically $300{ }^{\circ} \mathrm{C}$. Tin oxide gas sensors are preheated to facilitate the sensor's reaction before being exposed to an analyte gas, by the MHP. The baseline resistance of a tin oxide gas sensor is around one to several $\mathrm{M} \Omega$, and the resistance can be 10-1000 times lower than the baseline when reacting with target gases.

The monolithic tin oxide gas sensor array in this work was designed and fabricated using our in-house $5 \mu \mathrm{m}$ 1-metal, 1-poly CMOS process. The micrograph of the fabricated monolithic gas sensor array is shown in Figure 1. The sensor array consists of 16 micro-hotplate elements located in 4 rows and 4 columns.

The fabricated gas sensor array was tested under 4 different analyte gases which are Hydrogen, Ethanol, Carbon monoxide and Methane, respectively. All the test were performed at an operating temperature of $300{ }^{\circ} \mathrm{C}$. The responses to the 4 gases at different concentrations are shown in Figure $2 \mathrm{a}-\mathrm{d}$, respectively, where the output is the sensor's resistance.

\section{A SPIKING NEURAL NETWORK FOR ODOR DISCRIMINATION}

Our spiking neural network (SNN) requires two layers of neurons. First, the output of the gas sensors is transformed into a spike timing pattern by a layer of input neurons. Second, a layer of output neurons is trained with a supervised learning rule to categorize the spike timing patterns. Learning modifies the neural afferent synaptic weights of each output neuron to ensure that its potential will reach a threshold value for a set of incoming spike timing patterns and will not for another set. Each output neuron is expected to fire only in the presence of a given gas. The discrimination of four different gases, as in our application, will then require four output neurons (see Figure 3).

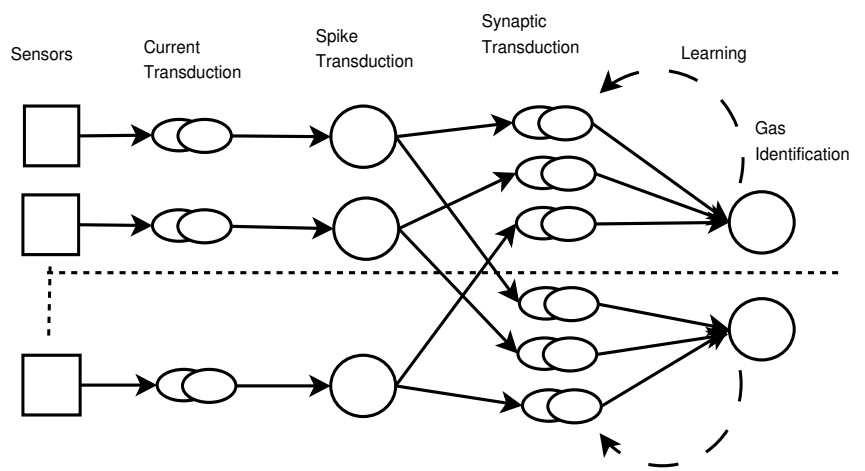

Fig. 3. Representation of the gas identification process. Each sensor's resistance is transduced into an electrical current which generates a neuron's spike. The spike pattern is transformed into synaptic currents which excites a second layer of neurons. Each of those neurons uses a learning rule to adapt its synaptic weights to respond to only one odor.

\section{A. Spike timing transduction by the input neurons}

The initial stage of the processing consists in the transduction from the gas sensors resistance into a neural spike timing. As a stimulation of the ORNs by an odor induces an excitatory synaptic current on OB or AL cells, a decrease of sensors resistances $R_{\text {sensor }}$ will generate an excitatory current $I_{\text {syn }}=V_{d c} / R_{\text {sensor }}$ (where $V_{d c}$ is a constant reference voltage) into the input neurons of our network.

We consider here the Leaky Integrate and Fire (LIF) model 
for the input neurons. Thus,

$$
C_{m} \frac{d V}{d t}=\frac{1}{R_{m}}\left(V_{\text {rest }}-V(t)\right)+I_{\text {syn }}
$$

where $C_{m}, R_{m}$ are the capacitance and resistance of the membrane and $V, V_{\text {rest }}$ are the membrane potential and the resting potential of the neuron, respectively.

By considering a constant sensor resistance, the time $T_{s}$ at which the neural potential reaches a threshold value $\theta$ is given by :

$$
T s=-\tau \ln \left(1-\frac{\theta}{I_{s y n} R_{m}}\right)
$$

where $\tau=R_{m} C_{m}$ is the membrane time constant.

As changes in the sensor resistances with respect to odor concentration is a parametric function $R(C)=$ $\left(R_{\text {sensor }_{1}}(C), \ldots, R_{\text {sensor }_{16}}(C)\right)$ where $\partial R_{\text {sensor }_{i}} / \partial C_{p p m} \leq$ 0 (see figure 2), changes in the spike timing is also a parametric function $T(C)=\left(T s_{1}(C), \ldots, T s_{n}(C)\right)$ where $\partial T s_{i} / \partial C_{p p m} \leq 0$ (see Figure 4 ).

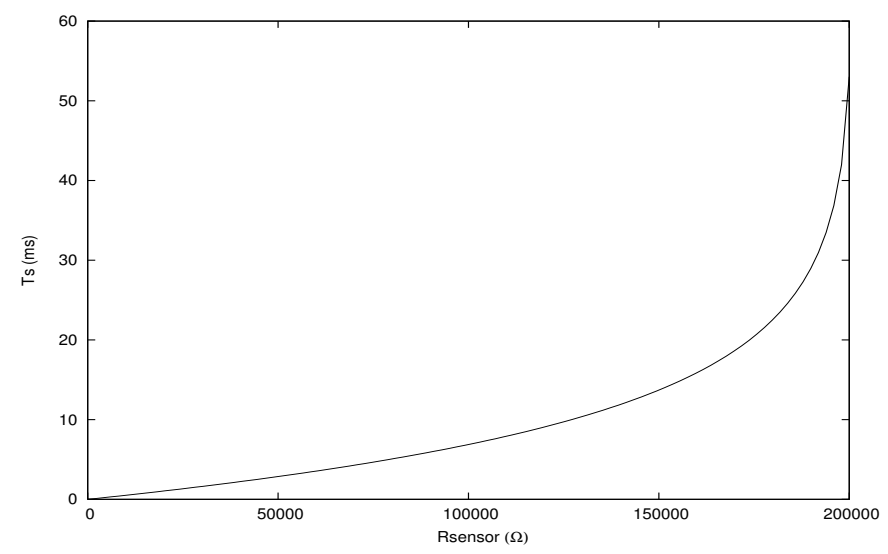

Fig. 4. Spike timing $T_{s}$ as a function of $R_{\text {sensor }}$. For $C_{p p m}=0, R_{\text {sensor }}$ is at its maximum $R_{0} \approx 200 \mathrm{k} \Omega$. When $C_{p p m} \rightarrow+\infty$ then $R_{\text {sensor }}$ reaches a saturation value $R_{\text {sat }}<<R_{0}$. Simulations have been performed with $V_{d c}=10 \mathrm{~V}, R_{m}=101 \mathrm{M} \Omega, C_{m}=99 \mathrm{pF}, \theta=0.02 \mathrm{~V}$.

\section{B. Odor discrimination by the output neurons}

Let us consider the following variable (for the $i^{\text {th }}$ input neuron) :

$$
\Delta_{T_{i}}= \begin{cases}t-T s_{i} & \text { if } t-T s_{i} \geq 0 \\ 0 & \text { if } t-T s_{i}<0\end{cases}
$$

One point $P_{(O, C)}\left(R_{\text {sensor }_{1}}, \ldots, R_{\text {sensor }_{16}}\right)$ corresponding to an odor $\mathrm{O}$ at a specific concentration $\mathrm{C}$ is transformed into a parametric function $f_{(O, C)}(t)=\left(\Delta_{T_{1}}, \ldots, \Delta_{T_{16}}\right)$. We call $\xi_{O}$ the set containing all the $f_{(O, C)}$ corresponding to every concentration $\mathrm{C}$ of one odor $\mathrm{O}$.

At the synaptic level, this information is transduced into post synaptic potentials (PSP) by a double exponential function :

$$
\begin{array}{r}
k: \Delta_{T_{i}} \rightarrow \exp \left(\frac{-\Delta_{T_{i}}}{\tau_{1}}\right)\left(1-\exp \left(\frac{-\Delta_{T_{i}}}{\tau_{2}}\right)\right) \\
P S P_{j}: \Delta_{T_{i}} \rightarrow w_{i j} \cdot k\left(\Delta_{T_{i}}\right)
\end{array}
$$

where $\tau_{1}$ and $\tau_{2}$ are parameters defining the shape of the PSP and $w_{i j}$ is the synaptic weight between the $i^{t h}$ neuron of the first layer and the $j^{\text {th }}$ neuron of the second layer.
Let us consider the $16^{t} h$ dimensional space $\Omega$ where each dimension i represents the synaptic activity of the first layer's neuron i. We call $K(t)=\left(k\left(\Delta_{T_{1}}\right), \ldots, k\left(\Delta_{T_{16}}\right)\right)$ the parametric function which represents the transformation due to $k$ on each $\Delta_{T_{i}} . F_{j}(t)=\left(P S P_{j}\left(\Delta_{T_{1}}\right), \ldots, P S P_{j}\left(\Delta_{T_{16}}\right)\right)$ is the parametric function which represents the transformation due to $P S P_{j}$ on each $\Delta_{T_{i}}$. For a reception of one $f_{(O, C)}(t)$, each neuron $\mathrm{j}$ of layer 2 generates the same $K(t)$ but different $F_{j}(t)$. By considering the diagonal matrix $W_{j}$ where each nonnull term of the column $\mathrm{i}$ is the value $w_{i j}$ we can write that $\forall t, K(t) . W_{j}=F_{j}(t) . W_{j}$ is thus a matrix of homothety in $\Omega$ transforming $K(t)$ into $F_{j}(t)$.

Each output neuron of the second layer sums all its incoming PSPs received over time and fires whenever the sum exceeds a given threshold value Vth (see Figure 5). In other words, the output neuron will fire if at least one point of the parametric function $F_{j}(t)$ belongs to the half-space defined by $\Theta \subset \Omega \mid \Sigma P S P_{j}\left(\Delta_{T_{i}}\right)>V t h$. Because $F_{j}(t)$ shape depends on $W_{j}$ whereas the threshold hyperplane stays unchanged, each output neuron $j$ has to find $W_{j}$ ensuring that it will reach $\Theta$ for one $\xi_{O}$ and not for the others to discriminate an odor.

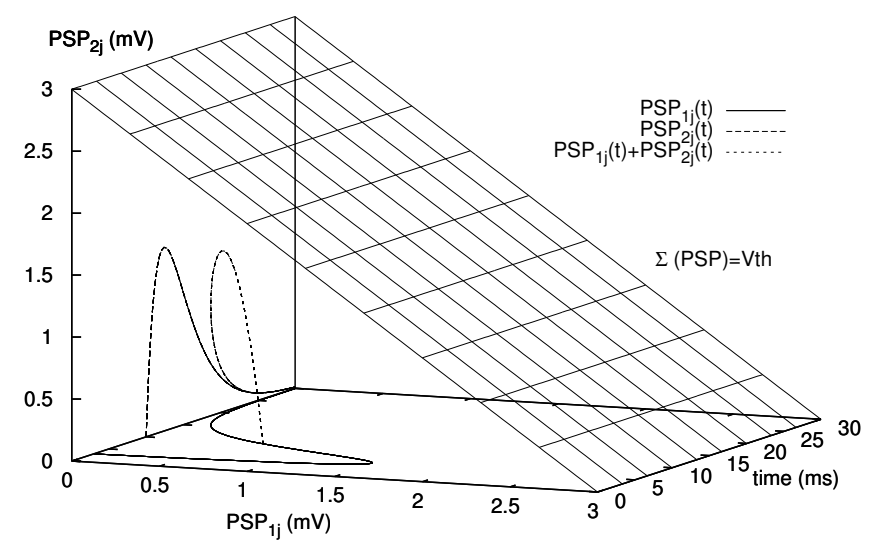

Fig. 5. Reception of 2 PSPs from different synapses as a function of time. The plan represents the threshold $\left(V_{t h}=3 \mathrm{mV}\right) . \tau_{1}=3 \mathrm{~ms}, \tau_{2}=10 \mathrm{~ms}$, $T s_{1}=3 m s, T s_{2}=10 m s, w_{1}=w_{2}=10$.

To find the weight matrix $W_{j}$ of the $j^{t h}$ output neuron, a supervised learning rule based on the work in [12] is used. The training set consists of spike patterns associated with their neural responses that should be attained. For a given training pattern, the neuron should respond or not. If the neuron responds whereas it should not or vice versa, an error is detected. The learning rule then modifies $W_{j}$ in order to minimize the error.

The following step is to find for which time called $T_{\text {error }}$, $F_{j}(t)$ is the deepest in $\Theta$ (resp. the closest to $\Theta$ ). Then the vector $\alpha . K\left(T_{\text {error }}\right)$, where alpha is an adjusting parameter, is decomposed in a diagonal matrix and substracted (resp. added) to $W_{j}$ (see Figure 6). This processus is repeated until no error is detected.

\section{EXPERIMENTAL RESULTS}

Data set collected from our in-house sensors using our experimented platform was used to generate a learning set (L). Linear interpolations from those records generate a new set of data used for testing the generalization performance type 1 generalization error (G1)-. By interchanging artificially 


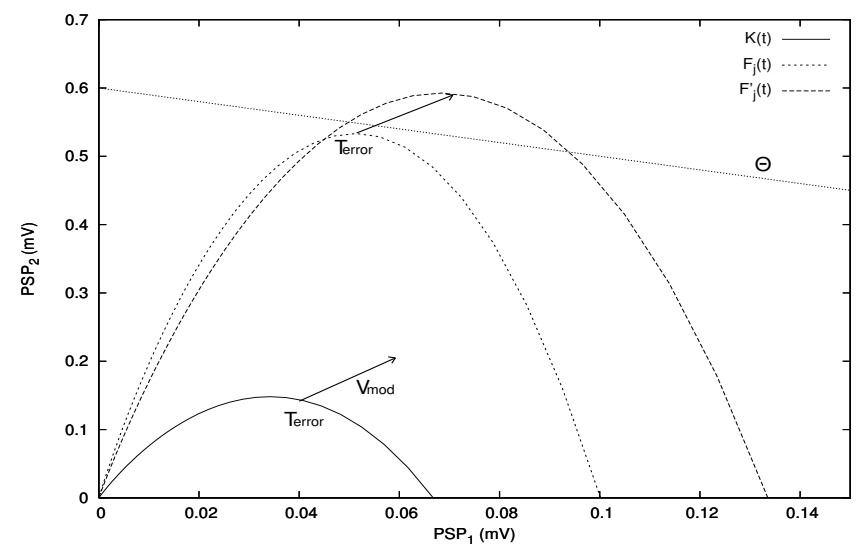

Fig. 6. Example of learning process. The curve $F_{j}(t)$ is the projection of $K(t)$ by the homothetic matric $W_{j}$. It has no points in $\Theta$ space whereas it should. The closest point to $\Theta$ occurs in $F_{j}(t)$ for $t=T_{\text {error }}$. The vector of modification $V_{\text {mod }}$ is defined by $\alpha . K\left(T_{\text {error }}\right)$. This vector is added to $W_{j}$ to generate a new projection $F_{j}^{\prime}(t)$ which presents some points inside $\Theta$.

the sensor responses from each odor and computing again a linear interpolation, a new set of data is generated. The error estimated from this new data set is called type 2 generalization error (G2).

As shown in Figure 7, the learning error rate decreases and reaches 0 at 370 training iterations. No modification in the weights are made afterwards. As a consequence, the generalization errors $\mathrm{G} 1$ and $\mathrm{G} 2$ do not change after iteration 370. Note that there is a strong correlation between the time evolution of the learning error rate and the generalization error rate $\mathrm{G} 1$. The generalization error rate $\mathrm{G} 2$ rapidly decreases at the beginning of the learning process and remains at a stable value afterwards.

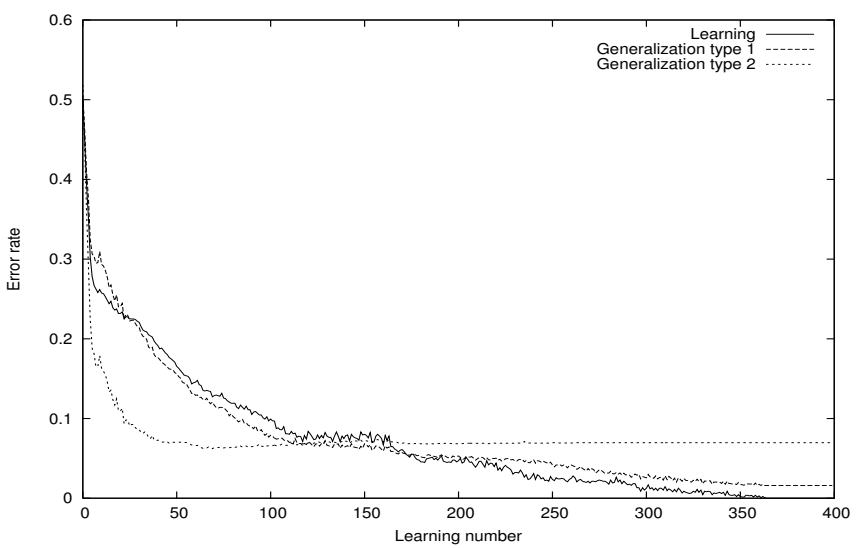

Fig. 7. Three error rates, for learning, generalization of type 1 and generalization of type 2 are presented as a function of the learning number. Error rate defines the ratio $N_{w} / N_{r}$ where $N_{w}$ is the sum of all the faulty neural responses and $N_{r}$ is the total of neural responses.

Table 1 reports the performance obtained with our spiking neural network as compared to the one obtained with a Support vector Machine (SVM). SVM(P1) stands for an SVM with a degree 1 polynomial kernel, $\operatorname{SVM}(\mathrm{P} 2)$ is for a degree 2 polynomial kernel and $\mathrm{SVM}(\mathrm{RBF})$ is for a radial basis kernel $(s t d=0.1)$. The SVM is trained directly on the normalized $R_{\text {sensors }}$ data. Table 1 shows that, for this discrimination task, our spiking neural network outperforms the SVM.
TABLE I

LEARNING AND GENERALIZATION PERFORMANCE FOR OUR SNN AND FOR SVM.

\begin{tabular}{|l|c|c|c|c|}
\hline & Our SNN & SVM(P1) & SVM(P2) & SVM(RBF) \\
\hline L & 0 & 0 & 0 & 0 \\
G1 & 0.016 & 0 & 0 & 0.097 \\
G2 & 0,07 & 0.251 & 0.243 & 0.017 \\
\hline
\end{tabular}

\section{CONCLUSION}

In this paper, a bio-mimetic algorithm based on spike timing computation for odor discrimination is demonstrated. It was shown that our bio-inspired algorithm outperforms a support vector machine when used in our in-house tin oxide gas identification application. Discriminating mixtures of gases probably needs additional neurons which will respond to a specific proportion of the different gases in the mixture. High gas concentrations result in low differentiation in the spike timing patterns where all the spikes are generated very early. A slow lateral inhibition, generated when synchronous spikes are detected in layer 1 , can lead to a decrease in the stimulations of the layer 1 neurons during the following cycle, increasing the relative spike time differences.

\section{ACKNOWLEDGMENTS}

The authors would like to acknowledge Prof. Gui-Zhen Yan and Professor Philip Chan for their considerable help on the gas sensor array integration.

\section{REFERENCES}

[1] S. Capone, A. Forleo, L. Francioso, R. Rella, P. Siciliano, and J. S. et al., "Solid state gas sensors: State of the art and future activities," Journal of Optoelectronics and Advanced Materials, vol. 5, pp. 1335-1348, 2003.

[2] P. Neaves and J. Hatfield, "A new generation of integrated electronic noses," Sensors and Actuators B, vol. 26-27, pp. 223-231, 1995.

[3] W. Goepel and K. Schierbaum, "Sno 2 sensors: Current status and future prospects," Sensors and Actuators B, vol. 26/27, pp. 1-12, 1995.

[4] P. M. Lledo, G. Gheusi, and J. D. Vincent, "Information processing in the mammalian olfactory system," Physiological Reviews, vol. 85 (1), pp. 281-317, 2005.

[5] G. Laurent, "A systems perspective on early olfactory coding," Science, vol. 286, pp. 723-728, 1999.

[6] P. Duchamp-Viret, M. Chaput, and A. Duchamp, "Odor response properties of rat olfactory receptor neurons," Science, vol. 284, pp. 2171-2174, june 1999.

[7] K. R. Illig and L. B. Haberly, "Odor-evoked acivity is spacially distributed in piriform cortex," Journal of Comparative Neurology, vol. 457, pp. 361-373, 2003.

[8] J. Perez-Orive, O. Mazor, G. C. Turner, S. Cassenauer, R. I. Wilson, and G. Laurent, "Oscillations and sparsening of odor representations in the mushroom body," Science, vol. 297, pp. 359-365, july 2002.

[9] S. Lagier, A. Carleton, and P. M. Lledo, "Interplay between local gabaergic interneurons and relay neurons generate $\gamma$ oscillations in the rat olfactory bulb," Journal of Neuroscience, vol. 24, pp. 4382-4392, may 2004.

[10] M. Ambard and D. Martinez, "Inhibitory control of spike timing precision," NeuroComputing, In press.

[11] K. J. Albert, N. S. Lewis, C. L. Schauer, G. A. Sotzing, S. E. Stitzel, and D. R. Walt, "Cross-reactive chemical sensor arrays," Chem. Rev., pp. 2595-2626, 2000.

[12] R. Gütig and H. Sompolinsky, "The tempotron: a neuron that learns spike timing-based decisions," Nature neuroscience, vol. 9, pp. 420428, march 2006. 